\title{
Postnatal Development of Metabolic Flexibility and Enhanced Oxidative Capacity After Prenatal Undernutrition
}

\author{
Amy M. Norman, MSc', Jennifer L. Miles-Chan, PhD $^{2}$, \\ Nichola M. Thompson, $\mathrm{PhD}^{3}$, Bernhard H. Breier, $\mathrm{PhD}^{4}$ and \\ Korinna Huber, Dr. ${ }^{5}$
}

\begin{abstract}
Metabolic flexibility is the body's ability to adapt to changing energy demand and nutrient supply. Maternal undernutrition causes growth restriction at birth and subsequent obesity development. Intriguingly, metabolic flexibility is maintained due to adaptations of muscle tissue. The aim of the present study was to investigate developmental pathways of these adaptive changes. Wistar rats received standard chow at either ad libitum (AD) or 30\% of ad libitum intake (UN) throughout pregnancy. At all ages, metabolic status indicated similar insulin sensitivity in $A D$ and $U N$ offspring despite the development of adiposity in UN offspring at weaning. Type IIA fiber size was reduced in soleus muscle of UN offspring at weaning and they had a higher percentage of type I fibers in adulthood with a concomitantly higher oxidative capacity. Plasticity of muscle was present during the postnatal period and proposes novel pathways for the dynamic development of metabolic flexibility throughout postnatal life.
\end{abstract}

\section{Keywords}

prenatal nutrition, postnatal development, muscle structure, metabolic flexibility

\section{Background}

Postnatal obesity is a well-described consequence of prenatal undernutrition in both humans and animals. ${ }^{1-6}$ However, recent research has shown that the impact of prenatal undernutrition on metabolic status varies according to the experimental model investigated. Using a rat model of prenatal undernutrition throughout pregnancy provided important insight into longterm consequences for the later metabolic status of offspring. The offspring of undernourished mothers developed obesity, hyperinsulinemia, and hypertriglyceridemia, but they remained insulin sensitive throughout adult life. ${ }^{7}$ In contrast to other experimental models of obesity, fat deposition was restricted to physiological fat depots and both muscle and liver glycogen levels were increased, indicating a physiological propensity to store energy. $^{7-9}$

The capacity of skeletal muscle to adapt its cellular regulation of fuel utilization to nutrient supply and demand is critical for the maintenance of metabolic health. The essential underlying metabolic features of skeletal muscle are influenced by multiple factors including structure, fiber-type composition, fiber size, mitochondrial density, innervation, and capillary density. Metabolism of type I fibers is mostly oxidative, while that of white type IIB fibers is almost glycolytic. Type IIA fibers are intermediary regarding oxidative and glycolytic capacity but have the highest capability to change their metabolic features due to external challenges such as exercise. The morphological and functional properties of muscle are determined during embryonic development and during the fetal and the early postnatal periods. It has been shown previously in both intrauterine growth restricted piglets and rodents that dietary influences, including calorie or protein restriction during the prenatal period, can change muscle development in terms of fiber number and fiber-type composition. ${ }^{4,10}$ In rats, skeletal muscle develops at distinct time points during both the prenatal and the early postnatal periods. While the majority of different muscle tissues in rats are developed during the early postnatal

\footnotetext{
I Department of Surgery, Faculty of Medical and Health Sciences, University of Auckland, Auckland, New Zealand

${ }^{2}$ Institute of Physiology, Department of Medicine, University of Fribourg, Fribourg, Switzerland

${ }^{3}$ Discipline of Physiology, School of Medical Sciences, Faculty of Health Sciences, The University of Adelaide, Adelaide, Australia

${ }^{4}$ Institute of Food, Nutrition and Human Health, Massey University, Albany Campus, Auckland, New Zealand

${ }^{5}$ Department of Physiology, University of Veterinary Medicine, Hannover, Germany
}

\section{Corresponding Author:}

Korinna Huber, Department of Physiology, University of Veterinary Medicine, Bischofsholer Damm 15/102, 30173 Hannover, Germany

Email: korinna.huber@tiho-hannover.de 
period (between birth and weaning), the number of fibers in some muscles, particularly the soleus (SOL), are fixed at the time of birth. ${ }^{11}$ In effect, skeletal muscle metabolism changes dynamically during this developmental period due to both intrinsic and extrinsic factors, such as nutrition and exercise. ${ }^{12,13}$ Poor fetal nutrition may reduce or delay skeletal muscle growth in order to favor neuronal development. ${ }^{14}$ In addition, dietary influences during pregnancy and lactation are known to have a strong impact on skeletal muscle differentiation and development as quantified by fiber number, fiber size, and fiber type. ${ }^{15}$ In a recent publication, we reported the impact of prenatal undernutrition on adult rat skeletal muscle morphology. The result manifested in adults as altered fiber-type composition which may reflect a state of enhanced metabolic flexibility during adult life. ${ }^{9}$

Metabolic flexibility is the body's ability to adapt to changing energy demand and nutrient supply. More specifically, it describes the capacity to effectively coordinate the regulation of fatty acids and glucose metabolic flux and is linked with insulin sensitivity. ${ }^{16}$ In contrast, a metabolically inflexible state is characterized by insulin resistance and impaired skeletal muscle fat oxidation. To date, the pathways to a metabolically flexible state, or the converse inflexible condition, are not well understood. However, there is increasing evidence that the oxidative capacity of skeletal muscle plays an important role in maintaining metabolic flexibility. ${ }^{17}$

While the impact of prenatal undernutrition is long lasting, the underlying developmental path for the observed adult metabolic status remains unclear. Therefore, the aim of the present study was to examine the postnatal development of muscle structure and function that are linked to metabolic fuel utilization. We hypothesized that skeletal muscle development adapts to reduced prenatal nutrition through structural and functional changes that favor pathways of flexible fuel usage and storage. We explored this hypothesis in prenatally undernourished rats by examining muscle morphology, fiber composition, and metabolic parameters at 3 developmentally important time points: birth, weaning, and adulthood. Structural and functional changes, especially an increased proportion of type I muscle fibers, together with an increased oxidative capacity of muscle tissue, may represent critical long-term changes that could explain increased metabolic flexibility during postnatal life.

\section{Methods}

\section{Experimental Design}

The breeding protocol used in this study was identical to that previously described. $^{7-9}$ Briefly, virgin Wistar rats were time mated and assigned to receive chow either ad libitum (AD, $\mathrm{n}=9$ ) or at $30 \%$ of ad libitum intake $(\mathrm{UN}, \mathrm{n}=9)$ throughout pregnancy. From the day of birth until weaning food was available to all dams. UN offspring were cross-fostered to AD fed mothers at day of birth to limit the nutritional influence to the prenatal period only. Distinct groups of $\mathrm{AD}$ and UN offspring were investigated at 3 developmental periods:
1. Neonatal $(\mathrm{N})$ : culled on the first postnatal day $(n=6$ per group, 12 in total),

2. Weaning (W): culled on postnatal day $21(\mathrm{n}=8$ per group, 16 in total),

3. Adult (A): culled on postnatal day $240(n=8$ per group, 16 in total).

Offspring of each group were from separate mothers to avoid litter effects. The first 2 groups, $\mathrm{N}$ and $\mathrm{W}$, included female offspring. Mixed-sex groups were studied as previous research identified no sex differences at 1 and 3 weeks of age in the parameters included in this project following maternal undernutrition. ${ }^{18}$ Importantly, muscle fiber-type composition is not affected by gender in AD and UN rat pups younger than 30 days. ${ }^{19}$ Male litter-mate pairs of group A were on a scheduled feeding regime of 2 hours (h) ad libitum access to food per day from 46 days (d) of age onward. The results of this adult group of animals have already been published elsewhere in another context. ${ }^{8,9}$ However, data from the adult group are included in the results of the present article to describe the development of structural and functional changes in muscle and of metabolic features. All procedures involving animals were carried out with the prior approval of the Animal Ethics Committee of the University of Auckland.

\section{Materials}

Analytical grade biochemicals were obtained from Merck (Darmstadt, Germany) or Sigma-Aldrich Inc (St Louis, Missouri) unless otherwise specified; reagents and apparatus for sodium dodecyl sulfate (SDS)-polyacrylamide gel electrophoresis and immunoblotting were from Bio-Rad (Hercules, California).

\section{Plasma and Tissue Collection}

Sample collection of adults was performed as described previously. ${ }^{8,9}$ In short, blood was collected from overnight-fasted rats into heparinized tubes and stored on ice until centrifugation. Plasma was stored at $-20^{\circ} \mathrm{C}$ until analysis. Liver samples were snap-frozen in liquid nitrogen and stored at $-80^{\circ} \mathrm{C}$ prior to analysis. Whole SOL and superficial gastrocnemius (GSUP) muscle from the right leg of offspring at weaning were also snap-frozen and stored at $-80^{\circ} \mathrm{C}$. The respective muscles from the left leg were prepared by fixing onto cork plates. Fixed muscles were frozen in liquid $\mathrm{N}_{2}$-cooled isopentane and stored at $-80^{\circ} \mathrm{C}$ to ensure tissue integrity for immunohistochemical studies. Due to size of SOL and GSUP samples in the N group the entire right hind whole leg muscle was snap-frozen. To ensure consistency of sample collection, the same investigator collected tissue samples of all animals.

\section{Immunohistochemical and Morphometrical Studies}

Skeletal muscle morphology and fiber-type composition was analyzed in offspring at weaning and at adulthood immunohistochemically as described previously. ${ }^{9}$ In each muscle, 
700 to 850 fibers per animal were counted and type-classified fibers were expressed as a percentage of total counted fibers. Size measurements of 50 randomly selected specifically immunostained fibers were made. In neonates, it was not possible to characterize respective fiber-type composition.

\section{Determination of Liver Glycogen Content and Protein Kinase $C \zeta$ Protein Expression}

Liver glycogen of offspring at weaning was determined according to Roehrig and Allred. ${ }^{20}$ For Western blotting, livers of all 3 groups were ground under liquid $\mathrm{N}_{2}$ and further homogenized on ice in prechilled homogenization buffer (in mmol/L: $50 \mathrm{~N}$ 2-hydroxyethylpiperazine- $N^{\prime}$-2-ethanesulfonic acid, $\mathrm{pH} 7.4$, $0.1 \%$ Triton X-100, 4 ethyleneglycol-bis(aminoethylether)tetraacetic acid, 10 EDTA, $100 \beta$-glycerophosphate, 15 tetrasodium pyrophosphate, 5 sodium orthovanadate, 25 sodium fluoride, protease inhibitors [Roche, Mannheim, Germany]); at a ratio of 30 to $50 \mathrm{mg}$ tissue powder per milliliter of buffer using an Eppendorf pestle and cup. Protein concentrations of the homogenates were measured according to Lowry method (BioRad protein quantification kit). Homogenates in loading buffer $(50 \mathrm{mmol} / \mathrm{L}$ Tris-HCl, pH 6.8, $10 \%$ glycerol, $2 \%$ SDS, $0.1 \%$ bromphenol blue, $2 \%$ mercaptoethanol) were denatured by incubation at $95^{\circ} \mathrm{C}$ for 5 minutes, separated by SDS gel electrophoresis with $40 \mu \mathrm{g}$ of protein loaded per lane and transferred onto nitrocellulose membranes according to Laemmli. ${ }^{21}$ Detection of protein kinase C (PKC) $\zeta$ (sc-216; Santa Cruz Biotechnology Inc, Santa Cruz, California) was performed after blocking the membranes in 10\% fat-free milk/PBST for 3 to 4 hours at room temperature (RT). Membranes were incubated overnight at $4{ }^{\circ} \mathrm{C}$ with rat-specific primary antibody. Detection of the primary antibody was performed by incubation with Sigma anti-rabbit-horse radish peroxidise (HRP) secondary antibody (A0545) for 1 hour at RT followed by incubation with enhanced chemiluminescence detection substrate (SuperSignal West Dura Extended Duration Substrate Kit). After band detection, expression was quantified by densitometry with Quantity One software (BioRad).

\section{Muscle Assays}

The muscle triglycerides (TGs) for the W and A groups only, and glycogen contents for all 3 groups were measured as described previously. ${ }^{20,7}$ Activity of citrate synthase (CS) and lactate dehydrogenase (LDH) were assayed in whole leg muscle homogenates for $\mathrm{N}$ cohort and in distinct SOL and GSUP tissues for the A and $\mathrm{W}$ groups. ${ }^{9}$ Briefly, muscle tissue was ground under liquid $\mathrm{N}_{2}$ and homogenized on ice in a prechilled homogenization buffer (50\% glycerol, in mmol/L: 40 $\mathrm{KCl}, 2$ EDTA, 25 Tris- $\mathrm{HCl}$ [pH 7.8] and 0.2\% Triton-X) using an Eppendorf pestle and cup. Enzyme activity was then quantified by spectrophotometric assay according to Newsholme and Crabtree ${ }^{22}$ (CS assay), and using a commercially available kit (Tox-7 toxicology assay kit, Sigma; LDH assay).

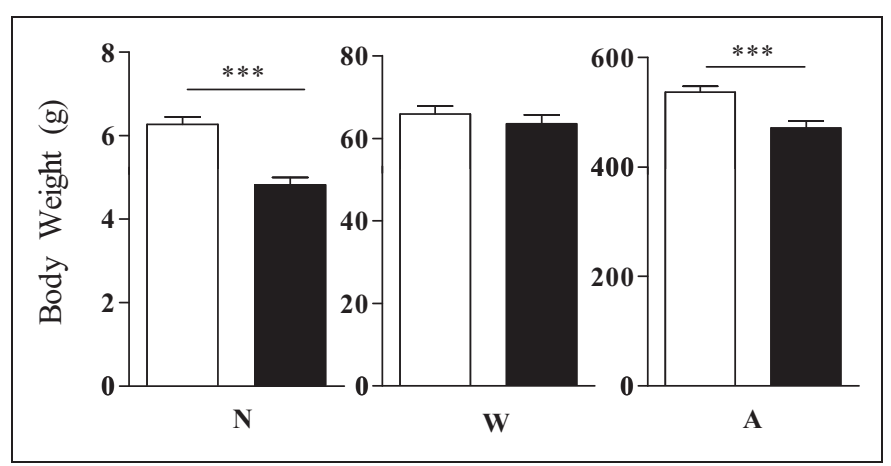

Figure I. Effect of maternal undernutrition during pregnancy on body weight in neonatal $(N)$, weaning $(W)$, and adult $(A) A D$ (offspring of ad libitum fed mothers; white bars) and UN (offspring of undernourished mothers; black bars) offspring (values are mean \pm standard error of the mean; $n=6-8)$. Maternal undernutrition during pregnancy induces significantly lighter offspring in the $N(* * * P<.00 I)$ and $A(* * * P<.00 I)$ groups. There is no significant effect of prenatal undernutrition on body weight in the $\mathrm{W}$ group.

\section{Plasma Metabolite and Hormone Assays}

Fasting plasma glucose and TG were measured by enzyme colorimetric assay using an automated bioanalyser (Roche/Hitachi 902; Roche Diagnostics, Penzberg, Germany). Total plasma leptin and C-peptide were measured using commercially available coated tube RIA kits (RL-83K and RCP-21K, respectively, Linco, St Charles, Missouri). Fasting plasma insulin levels were measured by enzyme-linked immunosorbent assay (110-1124 Mercodia AB, Uppsala, Sweden).

\section{Statistical Analyses}

Given that these animals are from different groups, statistical comparisons could only be made within each age group for AD versus UN offspring. Using the Kolmogorov-Smirnov test, we determined which data, in particular from the A and $\mathrm{W}$ groups, were compatible with a Gaussian distribution. The data that did not fit a normal distribution were subjected to nonparametric testing (Mann-Whitney $U$ test) where indicated. All other statistical significance of difference measures are estimated by Student $t$-test. Analyses were performed using GraphPad Prism (Version 5.0 for Windows; GraphPad Software, San Diego, California). Data are presented as \pm standard error of the mean, $\mathrm{n}=6$ to 10 animals per group. $P<.05$ was considered significant.

\section{Results}

\section{Postnatal Growth}

We have previously demonstrated that maternal undernutrition during pregnancy results in intrauterine growth restriction without a change in litter size..$^{7-9,23}$ In the present study, UN offspring weighed less than $\mathrm{AD}$ counterparts in the $\mathrm{N}$ and $\mathrm{A}$ groups. However, the $\mathrm{W}$ group UN offspring were comparable in body weight to the AD offspring (Figure 1). 


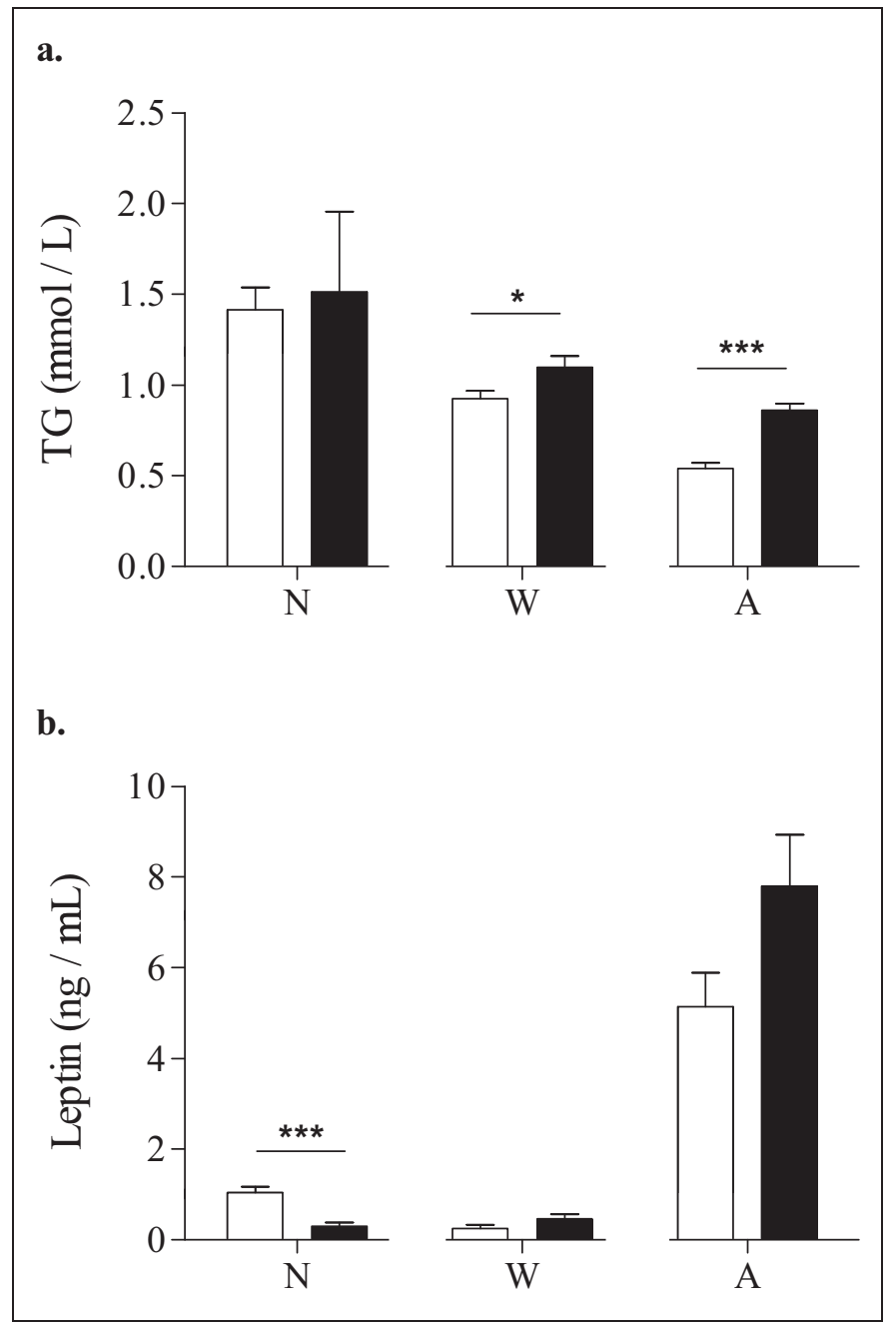

Figure 2. A, Effect of maternal undernutrition during pregnancy on triglyceride (TG) levels in plasma in neonatal $(N)$, weaning $(W)$, and adult (A) AD (offspring of ad libitum fed mothers; white bars) and UN (offspring of undernourished mothers; black bars) offspring (values are mean \pm standard error of the mean; $n=6-8$ ). Triglyceride levels are significantly elevated in the prenatally undernourished offspring compared to $A D$ offspring in the $W(* P<.05)$ and $A(* * * P<.001)$ groups. $B$, Effect of maternal undernutrition during pregnancy on leptin levels in plasma in neonatal $(N)$, weaning $(W)$, and adult (A) AD (white bars) and UN (black bars) offspring (values are mean \pm standard error of the mean; $n=6-8$ ). The leptin levels in UN offspring are significantly lower at birth $(\mathrm{N})$ than in $A D$ offspring $(* * * P<.0 \mathrm{I})$. In the A group, plasma levels tend toward hyperleptinemia in UN offspring $(P=.07)$. Comparing AD offspring at birth and weaning by $t$ test leptin levels decrease significantly $(P<.000 \mathrm{I})$, while leptin remains low in UN offspring from birth to weaning (not indicated in the figure).

\section{Measures of Lipid Metabolism}

Plasma TG levels showed a dynamic pattern in response to prenatal nutrition at the respective developmental ages. Plasma TG levels were comparable between AD and UN offspring at birth but increasingly elevated in UN offspring of both the $\mathrm{W}$ and $\mathrm{A}$ groups (Figure 2a).
Plasma leptin showed a dynamic pattern with differential outcomes of prenatal nutrition at each of the 3 developmental stages. Prenatal undernutrition resulted in reduced leptin levels as observed in the UN offspring of $\mathrm{N}$ group (Figure $2 \mathrm{~b}$ ). The plasma leptin levels of UN offspring were comparable to those of AD offspring at weaning and then displayed a tendency for hyperleptinemia at a mature age $(P=.07)$.

\section{Fasting Plasma Glucose, C-Peptide, and Insulin levels}

In $\mathrm{N}$ and $\mathrm{W}$ groups, no significant effects of prenatal undernutrition on plasma glucose were observed ( $\mathrm{N}$ glucose [in mmol/ $\mathrm{L}]$ : AD $3.99 \pm 0.17$, UN $5.16 \pm 2.26 ; \mathrm{W}$ glucose [in $\mathrm{mmol} / \mathrm{L}]$ : $\mathrm{AD} 5.28 \pm 0.34, \mathrm{UN} 4.88 \pm 0.70$ ); However, glucose levels in the A group showed a trend to higher levels in UN offspring (A glucose [in mmol/L]: AD $7.08 \pm 0.25, \mathrm{UN} 7.96 \pm 0.43$, $P=.093$ ). Plasma circulating insulin levels could not be measured for the $\mathrm{N}$ group owing to low blood volumes. Nevertheless, as C-peptide serves as a marker of insulin secretion from the pancreas, equal C-peptide levels indicated that there was no change in insulin secretion during the neonatal period for $\mathrm{UN}$ offspring (N C-peptide [in nmol/L]: AD $0.703 \pm 0.19$, UN $0.358 \pm 0.16)$. There was also no effect of prenatal undernutrition on either $\mathrm{C}$-peptide or insulin measures in the weaning period (W insulin $[\mathrm{ng} / \mathrm{mL}]$ : AD $0.16 \pm 0.01$, UN $0.17 \pm$ 0.01 ; W C-peptide $[\mathrm{nmol} / \mathrm{L}]:$ AD $0.18 \pm 0.02$, UN $0.16 \pm$ 0.02). At the adult age, mean levels of insulin and C-peptide were increased in UN offspring; however, this was not significant (A insulin $[\mathrm{ng} / \mathrm{mL}]$ : AD $0.63 \pm 0.08, \mathrm{UN} 1.22 \pm 0.35$; A C-peptide $[\mathrm{nmol} / \mathrm{L}]:$ AD $0.99 \pm 0.09, \mathrm{UN} 1.38 \pm 0.20)$.

\section{Hepatic Insulin Signaling}

The key insulin signaling protein in the liver, $\mathrm{PKC} \zeta$, was not significantly different at either the neonatal or weaning time points $(\mathrm{N}$ PKC $\zeta$ : $\mathrm{AD} 100.0 \pm 32.2, \mathrm{UN} 43.5 \pm 8.6 ; \mathrm{W}$ PKC $\zeta:$ AD $117.3 \pm 8.2$, UN $106.4 \pm 6.1$ ). However, in the adult cohort, expression of PKC $\zeta$ was significantly increased in UN offspring (A PKC $\zeta:$ AD $100.0 \pm 16.4$, UN $167.03 \pm$ $24.5 ; P<.05)$. The PKC $\zeta$ protein expression was paralleled by similar hepatic glycogen storage at weaning ( $\mathrm{W}$ glycogen $[\mathrm{mg} / \mathrm{g}] \mathrm{AD} 2.22 \pm 0.32$, UN $1.63 \pm 0.23)$ in addition to slightly, but not significantly, increased stores in adults (A glycogen $[\mathrm{mg} / \mathrm{g}]$ AD $8.53 \pm 1.21$, UN $11.84 \pm 1.21 ; P=.068)$. Measurement of glycogen in $\mathrm{N}$ livers was not possible due to small amounts of tissue.

\section{Skeletal Muscle Structure and Metabolic Function}

Energy Storage in Skeletal Muscle. In neonates, whole leg muscle of UN offspring showed lower concentrations of glycogen compared to AD offspring ( $\mathrm{N}$ muscle glycogen $[\mathrm{mg} / \mathrm{g}$ tissue] $\mathrm{AD} 1.14 \pm 0.11$, UN $1.05 \pm 0.08 ; P<.05)$. Both GSUP and SOL muscles at weaning showed no differences in glycogen content between $\mathrm{AD}$ and $\mathrm{UN}$ offspring (W muscle glycogen 
Table I. The Effect of Maternal Undernutrition During Pregnancy on Structure of SOL in Offspring at Weaning and Adulthood ${ }^{\mathrm{a}}$

\begin{tabular}{|c|c|c|c|c|c|}
\hline & \multirow{2}{*}{$\begin{array}{c}\text { Age } \\
\text { Prenatal Nutrition }\end{array}$} & \multicolumn{2}{|c|}{ W } & \multicolumn{2}{|c|}{ A } \\
\hline & & $A D$ & UN & $A D$ & UN \\
\hline \multirow[t]{2}{*}{ SOL, \% } & Type I & $64.12 \pm 1.66$ & $60.76 \pm 2.21$ & $92.70 \pm 1.62$ & $97.24 \pm 0.93 *$ \\
\hline & Type IIA & $46.38 \pm 3.56$ & $44.04 \pm 1.64$ & $6.629 \pm 1.71$ & $4.138 \pm 1.43 *$ \\
\hline \multirow[t]{2}{*}{ SOL, $\mu \mathrm{m}^{2}$} & I fiber size & $1040 \pm 22.90$ & $1067 \pm 46.73$ & $2287 \pm 109.8$ & $1971 \pm 89.64$ \\
\hline & IIA fiber size & $752.1 \pm 23.88$ & $667.1 \pm 27.07^{*}$ & $2110 \pm 101.40$ & $1570 \pm 163.8^{*}$ \\
\hline
\end{tabular}

Abbreviations: SOL, soleus muscle; AD, offspring of ad libitum fed mothers; UN, offspring of undernourished mothers; SEM, standard error of the mean.

${ }^{a}$ The effect of maternal undernutrition during pregnancy on structure of SOL in weaning (W), and adult (A) groups (values are mean $\pm S E M ; n=6-8$ ). Fiber size refers to the measured size $\left(\mu \mathrm{m}^{2}\right)$ of specifically immunostained fibers for either myosin type I or myosin type IIA, (\%) refers to percentage of type I or II fibers/ totally counted fibers.

$*=P<0.05$

Table 2. The Effect of Maternal Undernutrition During Pregnancy on the Structure of GSUP in Offspring at Weaning and Adulthood ${ }^{\mathrm{a}}$

\begin{tabular}{|c|c|c|c|c|c|}
\hline & \multirow{2}{*}{$\begin{array}{c}\text { Age } \\
\text { Prenatal Nutrition }\end{array}$} & \multicolumn{2}{|c|}{ W } & \multicolumn{2}{|c|}{$A$} \\
\hline & & $A D$ & UN & $A D$ & UN \\
\hline \multirow[t]{3}{*}{ GSUP, \% } & Type I & $13.02 \pm 0.94$ & $13.00 \pm 0.88$ & $26.00 \pm 2.05$ & $22.51 \pm 1.15$ \\
\hline & Type IIA & $53.78 \pm 1.51$ & $48.30 \pm 2.49$ & $34.86 \pm 3.14$ & $29.44 \pm 1.29$ \\
\hline & Type IIB & $41.87 \pm 3.63$ & $48.34 \pm 3.81$ & $48.53 \pm 2.44$ & $43.30 \pm 1.07$ \\
\hline \multirow[t]{3}{*}{ GSUP, $\mu \mathrm{m}^{2}$} & I fiber size & $653.8 \pm 84.91$ & $771.8 \pm 74.78$ & $3840 \pm 230.9$ & $3692 \pm 319.1$ \\
\hline & IIA fiber size & $555.2 \pm 53.57$ & $595.4 \pm 83.62$ & $3701 \pm 310.2$ & $2981 \pm 252.6$ \\
\hline & IIB fiber size & $801.0 \pm 36.14$ & $819.0 \pm 48.49$ & $4705 \pm 399.1$ & $3680 \pm 338.9$ \\
\hline
\end{tabular}

Abbreviations: GSUP, gastrocnemius muscle; AD, offspring of ad libitum fed mothers; UN, offspring of undernourished mothers; SEM, standard error of the mean. ${ }^{a}$ The effect of maternal undernutrition during pregnancy on structure of GSUP in weaning (W), and adult (A) groups (values are mean \pm SEM; $n=6-8$ ). Fiber size refers to the measured size $\left(\mu \mathrm{m}^{2}\right)$ of specifically immunostained fibers for either myosin type I or myosin type IIA, (\%) refers to percentage of type I or II fibers/totally counted fibers. A trend for lower type II fiber sizes was observed in adult UN GSUP muscle (type IIA $P=.0972$; type IIB $P=.070$ ).

$[\mathrm{mg} / \mathrm{g}$ tissue $] \mathrm{AD} 2.34 \pm 0.20, \mathrm{UN} 2.31 \pm 0.11$ ) nor was there any apparent difference in adult SOL glycogen content (A SOL glycogen $\mathrm{mg} / \mathrm{g}$ tissue AD $1.06 \pm 0.32$, UN $0.95 \pm$ $0.15)$. However, an increase in the glycogen content was observed in GSUP in the adult UN offspring (A GSUP glycogen $[\mathrm{mg} / \mathrm{g}$ tissue] AD $0.51 \pm 0.04$, UN $0.72 \pm 0.04 ; P<.01)$.

The TG content in mixed skeletal muscle was similar between $\mathrm{AD}$ and $\mathrm{UN}$ offspring at weaning and at adulthood (data not presented). The TG levels could not be assessed in neonates due to the small size of the muscle samples.

\section{Muscle Structure}

Accurate analysis of the neonate skeletal muscles was not possible due to the small size at birth. However, immunohistochemical staining for analysis of SOL and GSUP was performed at weaning and at adult age. Tables 1 and 2 summarize the fiber-type composition and fiber size of SOL and GSUP muscles. There are no differences in SOL type I or IIA fiber percentages per area in UN offspring compared to $\mathrm{AD}$ offspring at weaning. Furthermore, there were no differences in the percentage or morphology of GSUP type I, IIA, or IIB muscle fibers at weaning. However, at weaning, there was a significant decrease in the size of type IIA fibers in SOL of UN offspring (Table 1). At adult age, differences also became apparent in fiber composition of SOL of UN offspring. The percentage of type I fibers was significantly increased inversely proportional to the percentage of type IIA fibers. The GSUP did not show any significant differences between $\mathrm{AD}$ and $\mathrm{UN}$ offspring with respect to fiber-type proportions or size at weaning. However, a trend for lower type II fiber sizes was observed in GSUP of adult UN offspring (Table 2).

\section{Markers of Oxidative and Glycolytic Metabolism in Muscle}

Homogenates of whole leg muscle tissue were used for each individual animal from the $\mathrm{N}$ group because of the small size available. The metabolic marker enzymes CS (Figure 3a and 3c) and LDH (Figure $3 \mathrm{~b}$ and $3 \mathrm{~d}$ ) demonstrate no significant influence of prenatal undernutrition on these oxidative or glycolytic markers in skeletal muscle, respectively, at this age. In addition, no prenatal effect was observed on these key enzymes in either SOL or GSUP at weaning. However, in adult SOL, UN offspring had significantly increased CS activity in addition to decreased LDH activity. Although the GSUP of adult UN offspring showed a trend toward increased CS levels, prenatal undernutrition had no influence on either of these 2 marker enzymes. 


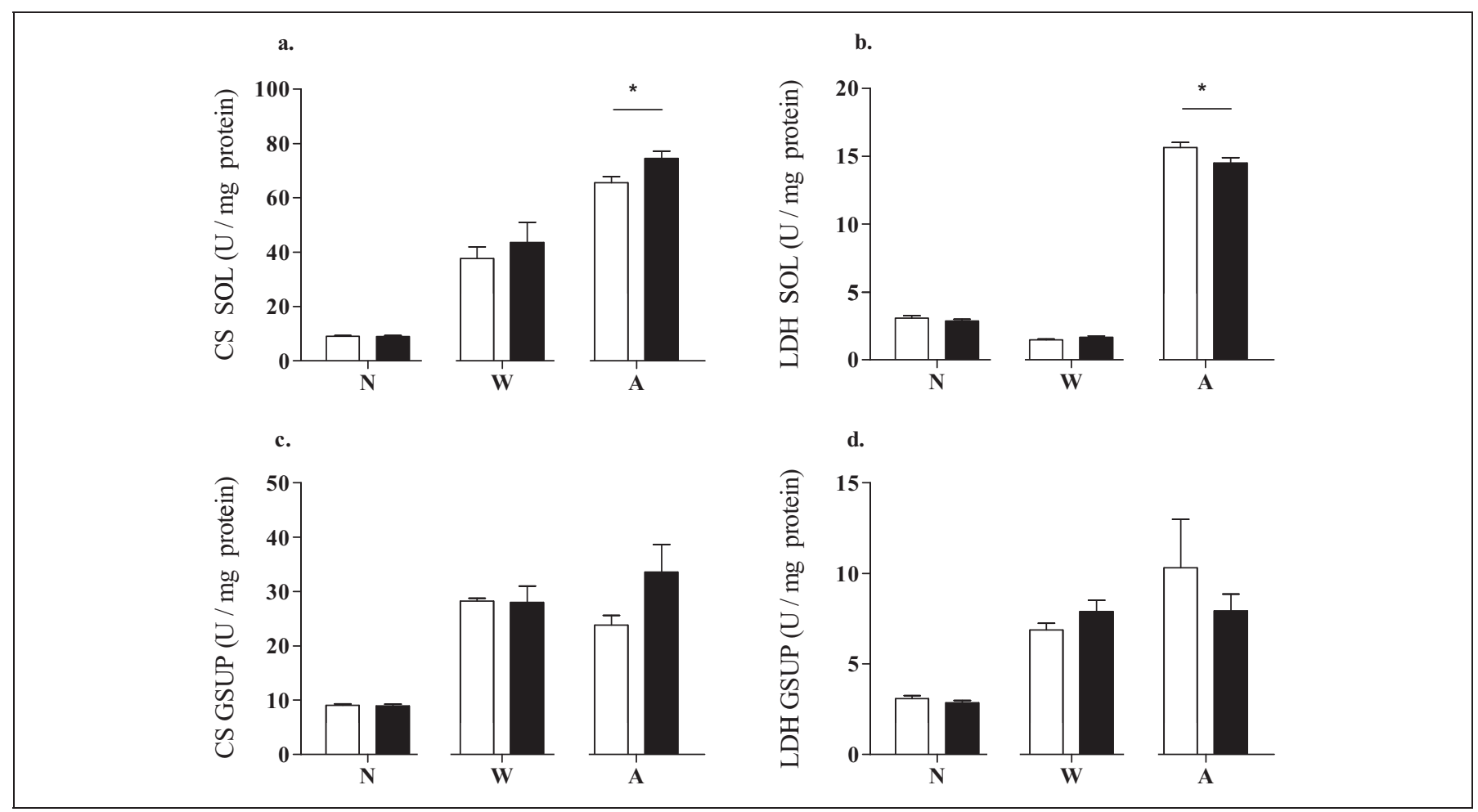

Figure 3. Effect of maternal undernutrition during pregnancy on oxidative and glycolytic enzyme activity in the SOL and GSUP (omit of offspring) in neonatal (N), weaning (W), and adult (A) AD (offspring of ad libitum fed mothers; white bars) and UN (offspring of undernourished mothers; black bars) offspring (values are mean \pm standard error of the mean, $n=6-8$ ); (A) SOL and (C) GSUP citrate synthase (CS; $\mathrm{mU} / \mathrm{mg}$ protein) for $A D$ and $U N$ offspring in N, W, and A groups. The activity of CS in SOL displays a difference only in the A group with a significant increase in UN SOL (*P<.05); (B) SOL and (D) GSUP lactate dehydrogenase (LDH; U/mg protein) for AD and UN offspring in N, W, and A groups. The activity of LDH in SOL displays a difference only in the A group with a significant decrease in UN SOL $(* P<.05)$ calculated by MannWhitney $U$ nonparametric test.

\section{Discussion}

Results of this study demonstrate that prenatal undernutrition sets in train structural and functional changes in muscle tissue, while their biological effects on metabolic fuel utilization manifest during the later postnatal period. Changes were induced before birth, becoming physiologically apparent at weaning. Higher TG concentrations in plasma may indicate that development of adiposity in UN offspring was initiated, while plasma variables of metabolic status suggest the presence of similar insulin sensitivity in $\mathrm{AD}$ and $\mathrm{UN}$ offspring at this developmental stage.

\section{Glucose Metabolism}

Lower glycogen stores in neonatal muscle of prenatally undernourished offspring may reflect the scarce energy supply during the prenatal period. Interestingly, this did not appear to influence other markers of glucose metabolism at birth such as plasma glucose, plasma C-peptide, liver glycogen, and liver PKC $\zeta$ levels, suggesting a successful adaptive outcome of energy metabolism during fetal development. By weaning, muscle glycogen stores in UN offspring increased to levels observed in $\mathrm{AD}$ offspring, indicating the onset of an increased capacity to store energy. Effective storage of energy at physiological sites is a key feature of metabolic flexibility. However, it is unclear if this increased energy storage is derived from a higher energy intake or a decreased energy expenditure of UN offspring during the suckling period. Further research is required to interrogate this question. In adult UN offspring, a small but consistent elevation in plasma glucose, insulin, and C-peptide concentrations and significantly higher expression of hepatic $\mathrm{PKC} \zeta$ and glycogen stores in muscle indicate a metabolic status of increased capacity to store energy as glycogen.

\section{Lipid Metabolism}

The birth weight difference between AD and UN offspring disappeared at weaning, indicating neonatal catch up growth in UN offspring. Elevated TG levels in UN offspring at weaning may herald the beginning of increased fat deposition which manifests as obesity later during adult life. In concurrence with this observation, plasma leptin levels, which were significantly lower in prenatally undernourished neonates, did not differ at weaning. As leptin is positively correlated with body weight at birth in rats and humans ${ }^{18,24}$ that difference in concentration at birth is not surprising as the UN offspring are smaller than the $\mathrm{AD}$ offspring. 


\section{Developmental Changes of Muscle Morphology and Oxidative Capacity}

In this study, muscle morphology and function, as assessed by immunohistochemical, biochemical, and enzymatic analyses, was predominately unchanged until weaning. Changes in fiber size and fiber-type composition began to emerge at weaning and were fully developed by adulthood. ${ }^{9}$ The specific reduction in the size of type IIA fibers in UN SOL at weaning demonstrates that prenatally induced pathways may initiate characteristic muscle morphological features at this age, although these early changes appeared to have no apparent functional consequences for oxidative and glycolytic capacity of muscle metabolism at weaning. By adulthood in UN, SOL morphology was characterized by a shift to more type I fibers at the expense of type II fibers that were reduced in number and size. ${ }^{9}$ Potentially, this increase reflects a shift to a more oxidative metabolic profile in prenatally undernourished offspring as indicated in the present study by increased oxidative CS and decreased glycolytic LDH enzyme activity. Type IIA fiber size was significantly reduced in SOL of adult UN offspring, as trend that was also observed in GSUP. While investigation of neonatal muscle morphology was not possible, this difference in fiber size was already apparent in SOL but not in GSUP at weaning and suggests that the growth and development of secondary fibers and progenitors of type II fibers in SOL muscle may already be reduced during early fetal development in UN offspring. The mechanism underlying the altered development of this muscle fiber morphology requires further investigation. Functionally, the decreased type IIA fiber size observed in UN offspring of the present study may facilitate the exchange of metabolic fuels more effectively. Given that these fibers have strong oxidative properties, the smaller type IIA fibers in UN offspring provides a greater surface area for gas exchange with the surrounding capillaries. ${ }^{25,26}$ Furthermore, the data presented here, coupled with the work of others, suggest that adaptation of skeletal muscle among other processes leading to the increased metabolic flexibility of prenatally undernourished offspring may be initiated by changes in plasma leptin levels during critical periods of development. ${ }^{27-30}$ The role of leptin in the regulation of skeletal muscle development has been illustrated by studies in pig primary cell cultures, where leptin promoted proliferation and inhibited differentiation of myoblasts. ${ }^{31}$ In the present study, plasma leptin concentrations were already altered by prenatal undernutrition on the first day after birth showing decreased leptin concentrations in UN offspring. Therefore, it is plausible to suggest that these altered patterns of circulating leptin may be linked with the observed changes in muscle fiber size and composition in UN offspring.

\section{Summary and Conclusion}

This study describes the apparent plasticity of skeletal muscle in rat offspring challenged by prenatal undernutrition and proposes novel pathways for the dynamic development of metabolic flexibility during postnatal life. In former studies, we have observed that increased metabolic flexibility is expressed even when prenatally undernourished offspring become obese as a result of exposure to a postnatal sedentary environment. ${ }^{7-9}$ We have identified that in UN rat offspring higher metabolic flexibility is based on the early development of an enhanced capacity to store energy in times of excess as glycogen and fat in physiological depots. While the metabolic profile showed changes commencing on the first day of postnatal life, specific features of muscle structure that facilitate effective utilization of metabolic fuels were slowly developed during postnatal development. The percentage of type I fiber expression and CS activity was increased in SOL muscle of adult UN offspring supporting a higher oxidative capacity of skeletal muscle. It is tempting to consider leptin as a key endocrine driver for differential muscle fiber development. Further investigations into the pathways responsible for stimulating type I fiber expression and potential selective atrophy of type IIA fibers are warranted. Higher oxidative capacity of skeletal muscle in UN offspring from the present study indicates that stored energy can be used effectively through activation of catabolic pathways in times of energy need. Stimulating these muscles by exercise postnatally was an efficient intervention to prevent obesity development in UN offspring. ${ }^{8}$ In principal, the high level in skeletal muscle plasticity in response to environmental stimuli is the reason why exercise intervention might be able to correct metabolic disturbances induced by prenatal nutrition. ${ }^{32}$

\section{Authors' Note}

$\mathrm{AN}, \mathrm{JM}$, and NT participated in the animal experiment and carried out the molecular biological and biochemical lab work. AN and $\mathrm{KH}$ carried out the immunohistochemical analysis of SOL muscle. NT, BB, and $\mathrm{KH}$ participated in the design of the study. BB led the design of the research program and obtained the funding support for this study. All authors participate in writing the manuscript. All authors read and approved the final manuscript. The work was done at the Department of Surgery, Faculty of Medical and Health Sciences, University of Auckland, Auckland 1142, New Zealand.

\section{Declaration of Conflicting Interests}

The authors declared no potential conflicts of interest with respect to the research, authorship, and/or publication of this article.

\section{Funding}

The authors disclosed receipt of the following financial support for the research, authorship, and/or publication of this article: funded by the Health Research Council of New Zealand and the National Research Centre for Growth and Development. Amy Norman was supported by the National Heart Foundation and Nga Pae o te Maramatanga. Korinna Huber was supported by Deutsche Forschungsgemeinschaft / Schaumann Foundation. Jennifer Miles-Chan was supported by the National Research Centre for Growth and Development.

\section{References}

1. Nathanielsz PW, Thornburg KL. Fetal programming: from gene to functional systems-an overview. J Physiol. 2003;547:(pt 1)3-4. 
2. McMillen IC, Adam CL, Mühlhäuser BS. Early origins of obesity: programming the appetite regulatory system. $J$ Physiol. 2005;565(pt 1):9-17.

3. Phillips DIW, Jones A. Fetal programming of autonomic and HPA function: do people who were small babies have enhanced stress responses. J Physiol. 2005;572(pt 1):45-50.

4. Sayer AA, Cooper C. Fetal programming of body composition and musculoskeletal development. Early Hum Dev. 2005;81(9): 735-744.

5. Symonds ME, Stephenson T, Gardner DS, Budge H. Long-term effects of nutritional programming of the embryo and fetus: mechanisms and critical windows. Reprod Fertil Dev. 2007; 19(1):53-63.

6. Green A, Rozance P, Limesand S. Consequences of a compromised intrauterine environment on islet function. $J$ Endocrinol. 2010; 205(3):211-224.

7. Thompson NM, Norman AM, Donkin SS, et al. Prenatal and postnatal pathways to obesity: different underlying mechanisms, different metabolic outcomes. Endocrinology. 2007;148(5):2345-2354.

8. Miles JL, Huber K, Thompson NM, Davison M, Breier BH. Moderate daily exercise activates metabolic flexibility to prevent prenatally induced obesity. Endocrinology. 2009;150(1):179-186.

9. Huber K, Miles JL, Norman AM, Thompson NM, Davison M, Breier BH. Prenatally induced changes in muscle structure and metabolic function facilitate exercise-induced obesity prevention. Endocrinology. 2009;150(9):4135-4144.

10. Bauer R, Gedrange T, Bauer K, Walter B. Intrauterine growth restriction induces increased capillary density and accelerated type I fiber maturation in newborn pig skeletal muscles. J Perinat Med. 2006;34(3):235-242.

11. Wilson SJ, Ross JJ, Harris AJ. A critical period for formation of secondary myotubes defined by prenatal undernourishment in rats. Development. 1988;102(4):815-821.

12. Pagel-Langenickel I, Bao J, Pang L, Sack MN. The role of mitochondria in the pathophysiology of skeletal muscle insulin resistance. Endocr Rev. 2010;31(1):25-51.

13. Punkt K, Naupert A, Asmussen G. Differentiation of rat skeletal muscle fibres during development and ageing. Acta Histochem. 2004;106(2):145-154.

14. Zhu MJ, Ford SP, Means WJ, Hess BW, Nathanielsz PW, Du M. Maternal nutrient restriction affects properties of skeletal muscle in offspring. $J$ Physiol. 2006;575(pt 1):241-250.

15. Bedi KS, Birzgalis AR, Mahon M, Smart JL, Wareham AC. Early life undernutrition in rats. 1. Quantitative histology of skeletal muscles from underfed young and refed adult animals. Br J Nutr. 1982; 47(3):417-431.

16. Storlien L, Oakes ND, Kelley DE. Metabolic flexibility. Proc Nutr Soc. 2004;63(2):363-368.

17. Phielix E, Mensink M. Type 2 diabetes mellitus and skeletal muscle metabolic function. Physiol Behav. 2008;94(2):252-258.

18. Desai M, Gayle D, Babu J, Ross MG. Programmed obesity in intrauterine growth-restricted newborns: modulation by newborn nutrition. Am J Physiol Regul Integr Comp Physiol. 2005;288(1): R91-R96.

19. Lane RH, Maclennan NK, Daood MJ, et al. IUGR alters postnatal rat skeletal muscle peroxisome proliferator-activated receptor-(gamma) coactivator 1 gene expression in a fibre-specific manner. Pediatric Res. 2003;53(6):994-1000.

20. Roehrig KL, Allred JB. Direct enzymatic procedure for the determination of liver glycogen. Anal Biochem. 1974;58(2): 414-421.

21. Laemmli UK. Cleavage of structural proteins during the assembly of the head of bacteriophage T4. Nature. 1970;227(5259): 680-685.

22. Newsholme EA, Crabtree B. Maximum catalytic activity of some key enzymes in provision of physiologically useful information about metabolic fluxes. J Exp Zool. 1986;239(2): 159-167.

23. Woodall SM, Breier BH, Johnston BM, Gluckman PD. A model of intrauterine growth retardation caused by chronic maternal undernutrition in the rat: effects on the somatotrophic axis and postnatal growth. J Endocrinol. 1996;150(2):231-242.

24. Jahan S, Zinnat R, Hassan Z, Biswas KB, Habib SH. Gender differences in serum leptin concentrations from umbilical cord blood of newborn infants born to nondiabetic, gestational diabetic and type-2 diabetic mothers. Int J Diabetes Dev Ctries. 2009;29(4):155-158.

25. Nakatani T, Nakashima T, Kita T, et al. Cell size and oxidative enzyme activity of different types of fibers in different regions of the rat plantaris and tibialis anterior muscles. Jpn J Physiol. 2000;50(4):413-418.

26. Lunde IG, Ekmark M, Rana ZA, Buonanno A, Gundersen K. PPARdelta expression is influenced by muscle activity and induces slow muscle properties in adult rat muscles after somatic gene transfer. $J$ Physiol. 2007;582(pt 3):1277-1287.

27. Krechowec SO, Vickers M, Gertler A, Breier BH. Prenatal influences on leptin sensitivity and susceptibility to dietinduced obesity. J Endocrinol. 2006;189(2):355-363.

28. Vickers MH. Developmental programming and adult obesity: the role of leptin. Curr Opin Endocrinol Diabetes Obes. 2007;14(1): 17-22.

29. Vickers MH, Gluckman PD, Coveny AH, et al. The effect of neonatal treatment on postnatal weight gain in male rats is dependent on maternal nutritional status during pregnancy. Endocrinology. 2008;149(4):1906-1913.

30. Briana DD, Malamitsi-Puchner A. Intrauterine growth restriction and adult disease: the role of adipocytokines. Eur J Endocrinol. 2009;160(3):337-347.

31. Yu T, Luo G, Zhang L, Wu J, Zhang H, Yang G. Leptin promotes proliferation and inhibits differentiation in porcine skeletal myoblasts. Biosci Biotechnol Biochem. 2008;72(1):13-21.

32. Rooney K, Ozanne SE. Maternal over-nutrition and offspring obesity predisposition: targets for preventative interventions. Int J Obesity. 2011;35(7):1-8. 